\title{
Le NGF fait son entrée dans le cancer du sein
}

La croissance des cellules de cancer du sein est réglée par différents facteurs de croissance qui stimulent ou inhibent leur prolifération, leur différenciation et leur migration $[1,2]$. Par exemple, l'EGF (epidermal growth factor), les IGF (insulin-like growth factors), les FGF (fibroblast growth factors) et le TGF- $\alpha$ (transforming growth factor$\alpha)$ stimulent la prolifération des cellules cancéreuses mammaires, alors que le MDGI (mammary derived growth factor inhibitor) et le TGF- $\beta$ (transforming growth factor- $\beta$ ) ralentissent leur multiplication. En fait, à l'exception du MDGI, ces molécules n'ont pas été identifiées sur la base de leur capacité de contrôler la multiplication des cellules de cancer du sein ; cette propriété n'a été mise en évidence que secondairement. Néanmoins, l'identification de nouvelles molécules capables de moduler la croissance des cellules mammaires constitue un élément important pour la compréhension de la cancérogenèse mammaire ainsi que pour la conception de stratégies innovantes contre cette maladie qui reste l'une des formes les plus fréquentes et redoutables de cancer.

\section{Les neurotrophines ne seraient pas que neurotrophiques}

Jusqu'à présent, les membres de la famille des facteurs de croissance appelés neurotrophines, dont le NGF (nerve growth factor) est le membre prototype, n'avaient pas été impliqués dans la cancérogenèse mammaire. Depuis sa mise en évidence par Rita Levi-Montalcini, le NGF a été largement étudié pour son activité stimulatrice de la survie et de la différenciation neuronale et la famille des neurotrophines est essentiellement connue pour ces propriétés neurotrophiques [3]. Il est maintenant bien établi que le NGF interagit avec ses cellules cibles par l'intermédiaire de deux catégories de sites de fixation membranaires : des récepteurs à activité tyrosine kinase correspondant au proto-oncogène TrkA (p140trk) et des récepteurs accessoires, sans activité tyrosine kinase, connus sous le nom de p75, dont la fonction précise est encore controversée [4-6]. Outre ses propriétés neurotrophiques, on a décrit quelques rares autres activités biologiques du NGF. Ainsi, ce facteur est chimiotactique pour les mélanocytes et des anticorps dirigés contre le NGF ou p75 inhibent la migration des cellules de Schwann. Le NGF a aussi été décrit comme stimulateur de la prolifération de quelques types cellulaires comme les cellules chromaffines et les lymphocytes. Par ailleurs, des anticorps bloquants anti-NGF peuvent inhiber la croissance des kératinocytes, suggérant que le NGF pourrait stimuler de façon autocrine et paracrine la croissance de ces cellules. La potentielle activité mitogène du NGF amène naturellement à se poser la question de son rôle éventuel dans les phénomènes de cancérisation. Jusqu'à présent, l'implication du NGF dans le cancer n'a été clairement démontrée que dans le cas des cellules de la prostate pour lesquelles la production de NGF stimule la capacité d'invasion des cellules cancéreuses. En outre, l'expression de p75 diminue dans les cellules malignes et métastatiques comparées aux cellules normales, suggérant que l'analyse des récepteurs du NGF pourrait présenter un intérêt pour le pronostic du cancer de la prostate [7].

\section{Le NGF, mitogène \\ des cellules cancéreuses du sein}

Nous avons tout récemment montré que le NGF est un puissant mitogène pour les cellules de cancer du sein [8]. Cette activité, jusqu'alors non décrite, se manifeste pour l'ensemble des lignées cellulaires étudiées (MCF7, MDA-MB-231, T47-D, BT-20), que leur croissance soit ou non hormonodépendante. Ainsi, le NGF peut être ajouté à la liste des facteurs de croissance capables de stimuler la croissance des cellules de cancer du sein.
La stimulation de la prolifération de ces cellules par le NGF nécessite l'activité tyrosine kinase des récepteurs p140trk et se traduit par l'activation de la voie des MAP-kinases. Nous avons également détecté la présence de p75 à la surface de l'ensemble des types cellulaires étudiés. Le schéma de réception/signalisation du NGF dans les cellules mammaires semble donc globalement correspondre à ce qui est généralement décrit dans le cas des cellules neuronales. Mais, ce qui est surprenant c'est que le NGF n'a pas d'activité mitogène pour les cellules épithéliales mammaires normales; à notre connaissance, il s'agit du premier cas rapporté de facteur de croissance ayant une action mitogène uniquement sur les cellules cancéreuses et pas sur les cellules normales de sein. Cela suggère que le NGF pourrait avoir une fonction importante dans le début et la progression des tumeurs mammaires. Les cellules normales exprimant les deux types de récepteurs p140Trk et $p 75$, on peut se poser la question de la raison de leur absence de réponse mitogène au NGF. Nous avons montré que le NGF ne déclenche pas l'activation de la voie des MAP-kinases dans les cellules normales comme le fait l'EGF ; ces cellules pourraient donc manquer d'un élément de transmission du signal entre p140Trk et les MAP-kinases, ou seraient soumises à un mécanisme particulier d'inhibition de cette transmission. Une étude plus précise de la structure de p140Trk ainsi que des premiers éléments du signal NGF dans les cellules mammaires sera nécessaire pour comprendre cette différence de sensibilité. On peut aussi envisager que la réponse des cellules normales à une stimulation par le NGF n'est pas une augmentation de leur prolifération. En effet, il est maintenant établi pour les cellules neuronales que le NGF peut intervenir dans le contrôle de la mort cellulaire, bien que le mécanisme de cet effet ne soit pas encore bien compris : au niveau des récepteurs, différentes hypothèses

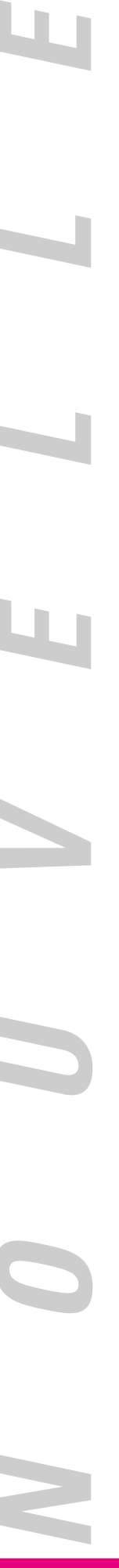

1125 
sont actuellement proposées qui impliquent p75 seul ou en combinaison avec Trk [4]. Cependant, nous n'avons pas mis en évidence d'effet du NGF sur la survie des cellules épithéliales mammaires normales. La migration de certains types cellulaires peut également être affectée par le NGF mais, là encore, aucune modification des capacités de migration des cellules mammaires n'a été mise en évidence après stimulation par le NGF.

L'effet du NGF sur les cellules épithéliales mammaires n'ayant pas été suspecté jusqu'à présent, l'expression de ce facteur et de ces récepteurs dans la glande mammaire n'a fait l'objet que de peu d'études. Les indications montrant la présence de NGF dans cet organe reposent essentiellement sur l'immunodétection de ce facteur dans le lait de souris [9] et, dans l'espèce humaine, une immunoréactivité anti-NGF rapportée au niveau d'implants mammaires [10]. Néanmoins, aucune de ces études n'a permis de préciser l'origine du NGF présent dans la glande mammaire (circulation générale ou production locale). Par ailleurs, il n'est pas exclu que le NGF présent dans la glande mammaire puisse également participer au développement et au maintien de l'innervation de cet organe.

En définitive, la découverte de l'action mitogène du NGF sur les cellules de cancer du sein pose un certain nombre de questions quant aux fonctions de cette molécule, et des neurotrophines d'une manière générale, dans la physiopathologie de la glande mammaire. Les travaux à venir, en permettant de mieux définir le rôle joué par le NGF dans la cancérogenèse du sein, devraient ouvrir de nouvelles perspectives pour la compréhension et éventuellement le traitement et le pronostic de cette maladie.

H.H.

X.L.B.

B.B.

S.D.

1. Ethier SP. Growth factor synthesis and human breast cancer progression. I Natl Cancer Inst 1995 ; 87: 964-73.

2. Rouayrenc JF. Les inhibiteurs de croissance des cellules mammaires. Med Sci 1997; 13: 89-92.

3. Lewin GR, Barde YA. Physiology of the neurotrophins. Annu Rev Neurosci 1996; 19: 289-317.

4. Bradshaw RA, Hondermarck H. Nerve growth
5. Lamballe F. Les récepteurs tyrosine kinases Trk : récepteurs de forte affinité des neurotrophines. Med Sci 1995; 11 : 1071-80.

6. Muller Y, Clos J. p75 : récepteur des neurotrophines. Med Sci 1997; 13: 978-86.

7. Pflug BR, Dionne C, Kaplan DR, Lynch J, Djakiew D. Expression of a Trk high affinity nerve growth factor receptor in the human prostate. Endocrinology 1995; 136 : 262-8.

8. Descamps S, Lebourhis X, Delehedde M, Boilly $B$, Hondermarck H. Nerve growth factor is mito- genic for cancerous but not normal human breast epithelial cells. J Biol Chem 1998; 273: 16659-62. 9. Grueters A, Lakshmanan J, Tarris R, Alm J, Fisher DA. Nerve growth factor in mouse milk during early lactation: lack of dependency on submaxillary salivary glands. Pediatr Res 1985; 19 : 934-7.

10. Lossing C, Hansson HA. Peptide growth factors and myofibroblasts in capsules around human breast implants. Plast Reconstr Surg 1993; $91: 1277-86$.

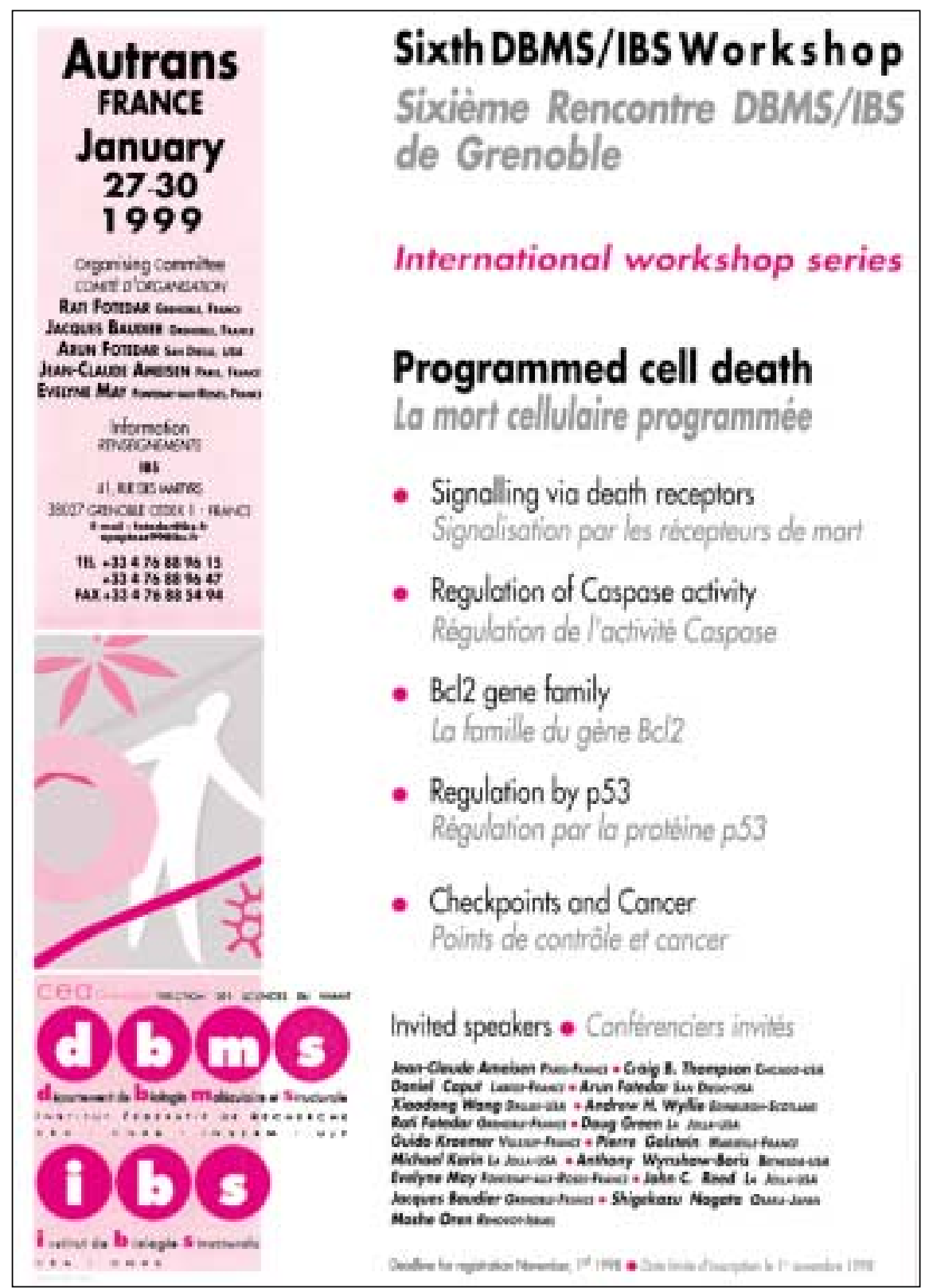

$\mathrm{m} / \mathrm{s} n^{\circ} 10$, vol. 14 , octobre 98 